\title{
Geographic dimensions of relevance
}

\author{
Stefano De Sabbata* \\ (University of Leicester, Leicester, UK) \\ s.desabbata@le.ac.uk \\ Stefano Mizzaro \\ (University of Udine, Udine, Italy) \\ mizzaro@uniud.it \\ Tumasch Reichenbacher \\ (University of Zurich, Zurich, Switzerland) \\ tumasch.reichenbacher@geo.uzh.ch
}

\begin{abstract}
Recent advances in mobile technologies led to new spatially-and context-aware definitions of relevance. In this paper, we aim to develop the conceptual framework suggested by Mizzaro (1998) further, in order to improve the characterization and classification of current and future concepts of relevance. We formalise two new dimension of relevance. The first dimension emphasizes the spatio-temporal nature of the information seeking process. The second dimension is necessary to describe how different concepts of relevance rely on different abstractions of reality.
\end{abstract}

\section{Introduction}

Almost a decade ago, Coppola et al. (2004) depicted what at the time was the raising mobile revolution, and its consequences in the field of Information Retrieval (IR). Mobile devices became ubiquitous, flooding our lives with a continuous stream of information, when the advent of social networks was still out of sight. Nowadays, prototypes such as those put forward by the Google's Project Glass ${ }^{1}$ outline a future in which (once again) "the nowadays complaints about having access to too much information will be seen with a small ironic grin and perhaps some nostalgia" (Coppola et al., 2004, p.1). Paraphrasing Coppola et al. (2004), "we can (still) hardly imagine what information overload is".

As mobile scenarios become more and more common, it is important to analyse how different relevance concepts deal with a user's mobility (Mountain, 2005; Mountain and MacFarlane, 2007; Raper, 2007; Raper et al., 2007), and represent the world surrounding a user (Reichenbacher, 2004; Reichenbacher and De Sabbata, 2011; De Sabbata and Reichenbacher, 2012). Relevance has always been recognized as a crucial notion for IR, and there is a vast body of literature devoted to it. Two studies are of particular interest for this paper, Mizzaro (1998), Coppola et al. (2004). Building on a previous proposal by Mizzaro (1998), Coppola et al. (2004) propose a framework to model the different concepts of relevance that can be considered in a mobile and context-aware scenario. The aim of this paper is to improve, modify, and extend the framework proposed by Mizzaro (1998) and Coppola et al. (2004), in order to account, more completely and precisely, for the characteristics of relevance concepts dealing with mobile information seeking scenarios. The proposed framework is intended as a tool to describe relevance concepts, and thus facilitate the classification and comparison of relevance concepts. It also aims at fostering the discussion and the development of information retrieval in the mobile and context-aware scenarios.

This paper is structured as follows. Section 2 briefly surveys the vast literature on relevance in IR and then describes the original framework introduced by Mizzaro (1998) and further developed by Coppola et al. (2004). In Sections 3 and 4, we present our extension to the original framework, and classify a set of concepts of relevance using the extended framework. Finally, Section 5 summarizes the discussion and concludes the paper.

${ }^{1}$ https://plus.google.com/+projectglass/posts, last accessed July 2013. 


\section{Background}

\subsection{Relevance in IR}

A comprehensive survey of the history of the concept of relevance in IR is out of the scope of this paper. The reader may refer to the well-known papers by Schamber et al. (1990), by Mizzaro (1997), and by Saracevic, who recently extended his seminal 1975 paper (Saracevic, 1975) with two new papers (Saracevic, 2007a; 2007b)

As suggested by Schamber et al. (1990), relevance is a multidimensional, dynamic, and complex concept. In information science, several efforts have been devoted since the 1960s to understanding its nature, and many definitions of relevance have been proposed. However, relevance remains an ill-defined concept, and the very nature of relevance rather implies the existence of different concepts of relevance (Borlund, 2003). Nevertheless, in IR, relevance is commonly defined as a relationship between a user's information need and an entity.

One of the most widely accepted definition of relevance has been proposed by Wilson (1973) as situational relevance. Situational relevance combines two relevance concepts. The first is logical relevance, as developed by Cooper (1971) more than forty years ago. Cooper defined logic relevance as "a relationship holding between pieces of stored information on the one hand and user's information needs formulated as information need representations on the other hand" (Cooper, 1971, p. 22). Cooper defined a user's question (i.e., a user's information need) as the sets of its possible answers. If at least one of those answers logically follows a piece of stored information, the latter is defined as logically relevant. This type of relevance is directly derived from deductive logic. The second concept included in situational relevance is evidential relevance. This is an inductive type of relevance, which conveys "the notion of degree of confirmation, or probability, of conclusions in relation to given premises" (Wilson, 1973, p. 460). This is an intrinsic factor of human knowledge and reasoning, and calls for the usage of plausible or probabilistic inference in IR. Hence, situational relevance is the relevance of a piece of information to the user's situation as she sees it, not as it 'really' is. The logical acceptation of relevance is the same embodied in logic relevance, but its clarity is 'infected' by the indeterminacy of evidential relevance.

\subsection{Mizzaro's framework}

Aiming to bring some order to the definitions of relevance, Mizzaro (1998) suggested four dimensions along which various types of relevance can be characterized. These dimensions are 'information resources' (i.e., what the user is searching for), 'user problem', 'time', and 'components'. The first dimension of this model contains three levels of abstraction: a document; a surrogate of the document (e.g., title, keywords, and abstract); and the information received by the user, as she perceives it. The user problem (second dimension) has four levels of abstraction: her real information need; the information need as she perceived it; the information need as she can express it in natural language; and the query as it is expressed in the system language. The third dimension 'time' refers to the steps in which the information flows with the interaction from the moment in which the user's real information need arises to the moment in which it is satisfied. The fourth dimension 'components' lists the aspects that compose the first two dimensions: the topic the user is interested in; the task or activity she aims to perform; and the context in which everything happens. In Mizzaro's framework, Wilson's situational relevance (Wilson, 1973) can be defined as the relevance of the information that the user receives to the perceived information need; Wilson's definition of situational relevance does not address the dimensions 'time' and 'components'.

\subsection{Coppola et al.'s framework}

Mizzaro's framework was further developed by Coppola et al. (2004) to define w-relevance, i.e., a notion of relevance adequate for the mobile world. The authors use the ' $w$ ' to refer to wireless relevance, but also double-relevance, world-relevance, and double-user-relevance (Coppola et al., 2004, p. 7). One of the changes in this new framework is that, in the first dimension, relevance can deal with the resources at four levels of abstraction: besides surrogate, document, and information, the actual entity (or thing) is added. W-relevance encapsulates a relevance addressing real/physical world entities and not only documents. It is also proposed to rename the dimension into 'resources'. A similar change is suggested on the second dimension, where 'thing need' is added. 
These further levels of abstraction are a key change, because they reveal a whole new layer of relevance concepts, beyond the classic horizon of document-based IR. These relevance concepts address the physical objects that are at the core of current e-commerce systems, recommender systems, and location-based services, that cannot be said to simply deal with the digital documents that describe and refer to physical objects. For example, if a user is searching for a restaurant for lunch, a webpage containing information about local restaurants might be considered relevant, although some of the restaurants described in the webpage might be not relevant, for example, because they are closed.

Other important changes to the original framework of Mizzaro (1998) applied by Coppola et al. (2004) concern the increased importance of physical world aspects, like time (third dimension) and location and other contextual aspects (fourth dimension). Indeed, the new framework seems more adequate to the mobile use case than the previous one; however, in our opinion, the framework can still be improved and generalized in several respects, as described in the following.

\section{Geographic dimensions of relevance}

In the previous section, we introduced the framework proposed by Mizzaro (1998) to characterise different types of relevance and the later extended framework suggested by Coppola et al. (2004) to include physical world entities (beyond documents that describe them) among the type of resources that concepts of relevance deal with. Although Coppola et al.'s changes are a step in the right direction, we believe that their framework still misses some important aspects of the notion of relevance implied by users' mobility, as users are situated in a geographic context in the real world and are seeking information with their mobile device.

In this section, we propose and discuss four main changes to Coppola et al.'s framework (2004), which hopefully will enable the framework to better deal with spatial and contextual notions of relevance. The first change relates to the first dimension of the framework, the second change to user's mobility, the third change to perceptions and representations of reality, and the fourth change to the components dimension. We discuss each of them in more detail in the following four subsections.

\subsection{Clarifications on the 'resources' dimension}

Coppola et al. (2004) added to the first dimension the new level of abstraction 'thing', and took into account the case of a document (e.g., a museum web page) referring to a thing (the real museum). However, they did not explicitly remark that, besides the document, the surrogate as well can refer directly to a thing, rather than refer to a document that then refers to a thing. For example, an entry in a land register, or an entry in any structured database, can hardly be considered as a document and would be more naturally interpreted as a surrogate. However, there is no corresponding document, since those entries refer directly to a 'thing': they can be understood as surrogates of things.

In an attempt to avoid confusion, in the following we will refer to a real-word thing (excluding documents) using the term 'entity', and the term 'document' will be used to refer to any kind of object containing (usually unstructured) human knowledge. We will refer to a structured representation of human knowledge (e.g., entries in a library database) using the term 'surrogate', as it is usually done in IR and library science. Finally, we will refer to a structured representation of real-world objects (e.g., entries in a land register) using the term 'feature', as it is commonly used in geographic information systems ${ }^{2}$ (although we use the term in a broader sense, assuming it can refer to any real-world object).

\subsection{From time to space-time}

Mizzaro (1998) describes how the relevance of a document (or any other abstraction level of resources) to a query (or any other abstraction level of information need) changes over time, as the user acquires information, and thus her information need changes. In this section, we argue that space plays now an important role in a mobile scenario, and in doing so, we also provide an example of a scenario where the resource whose relevance its being evaluated is an entity (i.e., a real-world thing, in the specific case, a restaurant) rather than a document.

The effect of time on relevance is captured in the framework by means of the dimension 'time', that allows to specify which moment of the information seeking process is taken into account by a relevance concept.

2 http://en.wikipedia.org/wiki/Feature_data, last accessed July 2013 
However, this dimension has been suggested accounting for a sort of stationary information seeking scenario, i.e., a scenario where a user is supposed to be sitting at a desk. This 'stationary' assumption does not hold anymore, as users can now search for information on their mobile devices. In fact, as discussed in time geography (see e.g., Miller and Bridwell, 2009), a mobile user can 'trade' time for space in order to generate mobility: the user can spend time travelling, obtaining in return the movement from a location to another (i.e., mobility). A user's location can change as time passes by from the moment the real information need arises until it is satisfied, and location can be a fundamental factor of relevance (Reichenbacher and De Sabbata, 2011): a piece of information which is relevant for the location where the user is when she perceives her information need may no longer be relevant for the location where she is when she obtains the retrieved information, because of the change in location.

For example, let us assume that a person is in a foreign city, walking from her hotel to a coach station, heading home. As she is a bit early and it is lunchtime, she decides to search for a restaurant on her smartphone, while continuing to walk. In this scenario, the query is something like 'restaurant', yet the entities to be retrieved are not documents (possibly about restaurants) to be read but objects referring to real restaurants in the physical world (e.g. points of interest on a digital map). It is important to note that a document containing information about restaurants in the area would be very relevant, even if one of the described restaurant is not, for instance because it is currently closed. This situation is represented in Figure 1: the user decides to have lunch when she is at location 1 , and makes her decision on where to go a few instants later, when she has walked until location 2 (let's say five or ten minutes later). In general, if the relevance of the three restaurants represented in Figure 1 is assessed when the user is at location 1, all three restaurants $\mathrm{A}, \mathrm{B}$, and $\mathrm{C}$ would have similar relevance in terms of spatio-temporal proximity. All of them are on the way to the coach station, and the user would be able to spend enough time at their location to accomplish her activity (i.e., having lunch), and then reach the coach station in time.

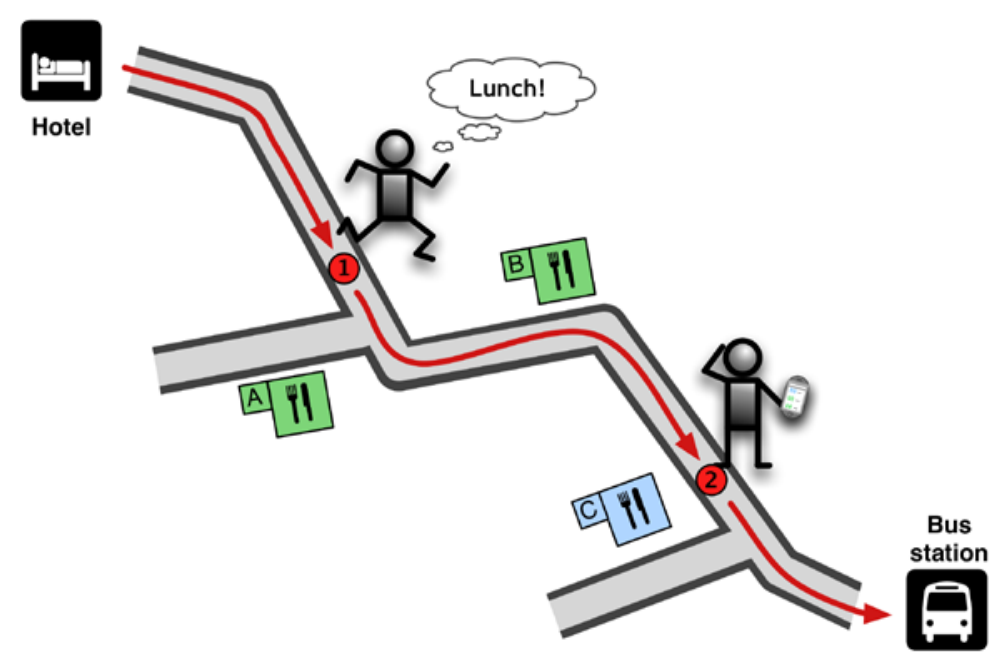

Figure1. Example of a user searching for a restaurant, while walking to the coach station.

This example can be further analysed by using the space-time prism (Hägerstraand, 1970; Miller, 1991), a concept from time geography similar to the well known phase space in mathematics and physics. Space is represented as bi-dimensional on the $\mathrm{x}$ and $\mathrm{y}$ axes, and time is represented by the $\mathrm{z}$ axis. The left part of Figure 2 shows such a space-time prism, whose meaning is as follows. As time flows bottom up, the prism delimits all the space that can be reached by the user within the time interval, given the constraint of being at the coach station on time (and her maximum travel speed). The red lines originating from the red circle labelled 1 represent the paths through space and time that the user can follow to reach the three restaurants A, B (green circles), or C (blue circle). Green and blue vertical lines represent the time the user can spend at the different locations (restaurants) after having followed one of the red lines (representing the corresponding space-time path). These vertical lines are parallel to the time axis as the user would not move as the time flows. The longer the line, the more time the user can spend at the location. The upper red lines converging to the grey circle represent the paths through space and time that the user would take in order to 
each the coach station. At the time the user is at location 1 all three green and blue lines are almost equally long, as the user's path to her destination passes by all three restaurants, thus the time left for lunch would almost be the same, whichever restaurants she chooses.

The space-time prism shown on the right side in Figure 2 represents the situation when the user is at location 2 and is considerably smaller, because time has passed as the user was walking and considering her options. By the time the user is at location 2, she has less time left to reach her destination, and thus only shorter distances can be covered. Moreover, walking backwards toward options A and B would mean to cover that distance again after lunch. Hence, if the relevance of the three restaurants is assessed when the user is at location 2 , the restaurant $\mathrm{C}$ would be more relevant in terms of spatio-temporal proximity, as the user would not need to walk back and then walk again toward location 2 and the coach station. Indeed, in the example on the right side in Figure 2, the two restaurants A and B are out of reach for time constraints and out of the space-time prism, thus absolutely not relevant. This scenario is not only theoretical. De Sabbata and Reichenbacher (2012) have shown that in similar situations users would consider restaurants A and B as less relevant than $\mathrm{C}$, because they are in the opposite direction from the final destination, even having enough time to pursue the desired activity.
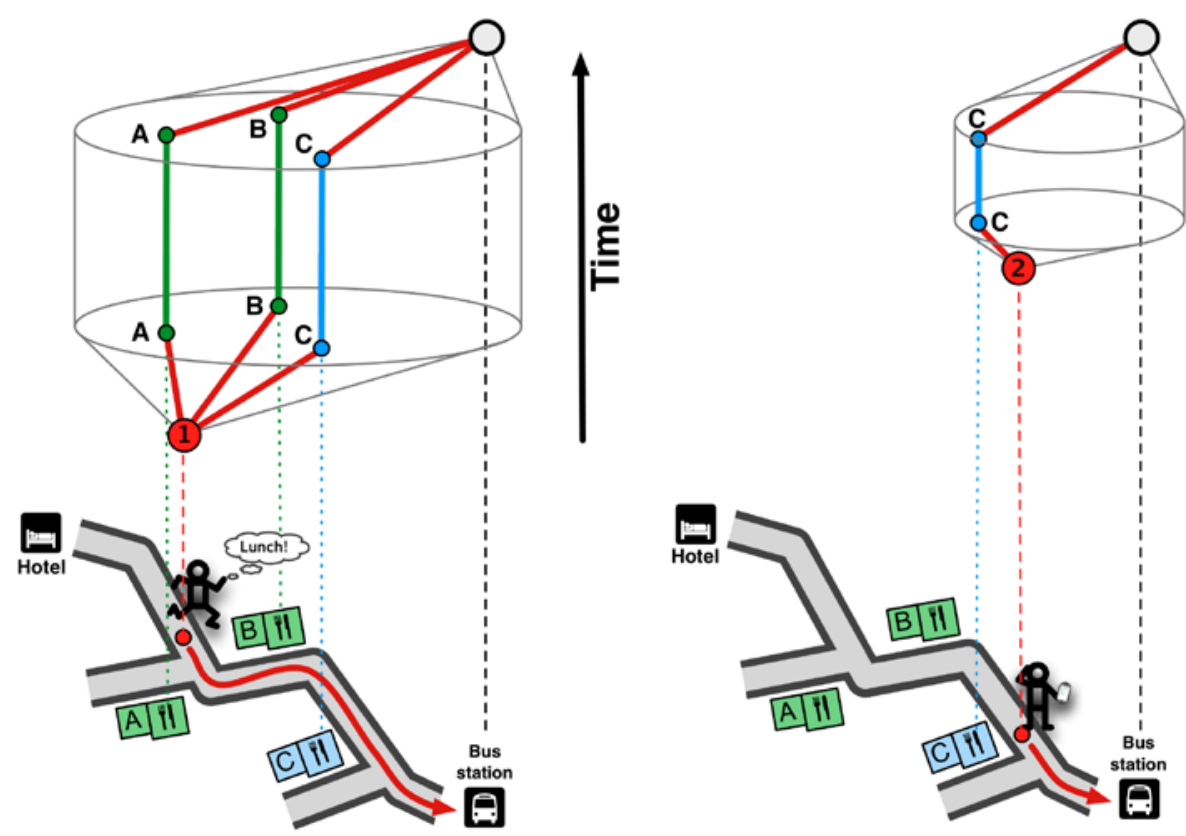

Figure2. Space-time prisms describing the user's mobility at locations 1 and 2 in Figure 1.

Therefore, there is a difference between relevance at the moment the real information need arises and relevance at the moment the information need is satisfied. This is already modelled in the dimension 'time' of Mizzaro's and Coppola et al.'s frameworks, but the difference of relevance in this example does not relate to changes in user's knowledge over time; rather, it relates to changes in the user's location in space over time, and therefore to mobility. In fact, if the user stands still at location 1 for five-ten minutes, the situation would be different and the three restaurants would have similar relevance. Also, if the user was able to teleport from location 1 to location 2, with no time lapse and no other modification of the situation, the relevance would still differ in the two locations.

Hence, a relevance dimension concerning time cannot ignore space, as these two factors are interdependent. Therefore, we suggest replacing the third dimension 'time' with a dimension concerning space-time. This new dimension 'space-time' describes how the information flows with the interaction, from the point in space and time in which the user is at the moment her real information need arises, to the point in space and time in which the user is at the moment the information need is satisfied.

In the example above, we used a very simple bi-dimensional model of space. However, the mobility and context components of relevance may require more sophisticated models of space in order to represent more complex geographic concepts (Golledge, 2002), such as spatial relationships and geographic associations (e.g., 
neighbourhood relationships, clusters, co-locations, etc.). Raper (2007) distinguishes two geographic models of space: a geo-representation (i.e., a space modelled with geometric entities), and a geo-context (i.e., a mental constructs, such as places or landmarks). According to Reichenbacher (2009), four different models of space can be defined: geometrical, topological, structural, and semantic (see Figure 3). The four models are characterized by increasing complexity, richness, and expressive power: the geometrical one includes metrics, the topological one adds relations, the structural adds configuration, and the semantic adds meaning.

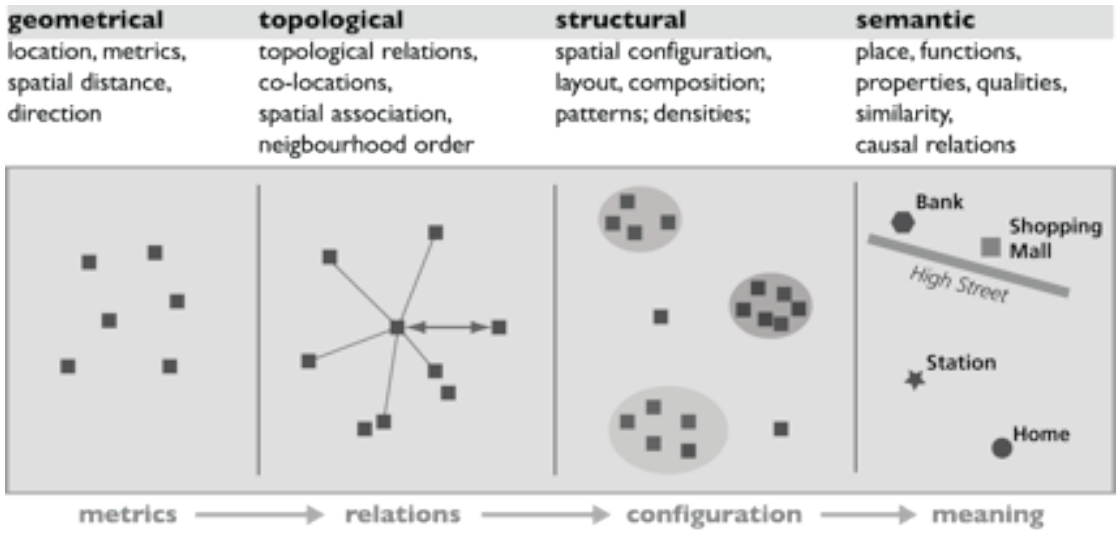

Figure 3. Different concepts of location and space (from Reichenbacher, 2009).

To show how these more sophisticated models of space can be used, one might consider that a structural conception of space is necessary to implement criteria of geographic relevance, such as cluster and colocation (De Sabbata and Reichenbacher, 2012). However, only a semantic conception of space would enable a comprehensive development of the activity, preferences, social, and context component, as it accounts for the functions, properties, and qualities of a place. For example, a label 'cafe' attached to a coordinate pair is not sufficient to encompass the essence of a place as it is experienced by it attenders. Further semantic information about the place and its surroundings is required to fully understand its affordances and how they change over time. A user's experience in a 'Starbucks' in New York at 8 a.m. would not be quite the same as in a 'Bar Sport' in a small Italian village at 6 p.m., although they both serve coffee.

Furthermore, it has to be noted that the dimension 'space-time' is used to define which user location in space and time is considered in assessing relevance, and it does not relate to the information need or information content. For instance, the geographic information retrieval systems discussed by Palacio et al. (2010) considers the spatial and temporal content of documents (i.e., as part of the topic the user is interested in, within the resource dimension), but not the spatial and temporal context in which the information seeking happens (i.e., the 'spacetime' dimension). For example, a user might be interested in 'treaties signed by Napoleon in Italy in the 1790s'. In such a case, the spatial and temporal facets concern the topic of interest, and they are not part of the user's context.

\subsection{A new dimension: 'world'}

Just as the real information need is different from the query received by the system, the real world is different from the world perceived by the system. This distinction is absent in Coppola et al.'s framework; hence, we propose to consider a new dimension to handle the representation of the world in which the relevance relation between the user's information need and the resource takes place.

A relevance concept can be described as dealing with reality at the following levels of abstraction:

- real world, which includes everything which is considered to be part of reality;

- documented world, that is the world as it is recorded by the human knowledge in any form of stored information;

- perceived world, that is, the world as known and perceived by a user;

- $\quad$ system world, that is, the world as the systems knows it (i.e., the data available both from resources of the system and sensors of the user's devices). 


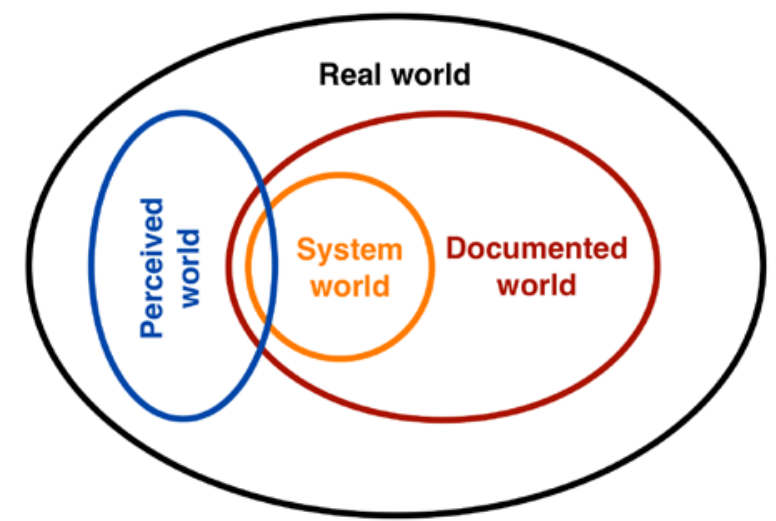

Figure 4. Levels of abstraction described by the 'world' dimension.

Figure 4 illustrates the mutual relationships between the four levels of abstraction described above, in the form of a Venn diagram. The all-encompassing set labelled "real world" contains the remaining three levels of abstraction. The "system world" is a subset of the "documented world. The "perceived world" includes part of the documented world and of the system world, as well as other parts of the real world - that is, that parts of reality that the user perceived, but which are not documented by the system or in any other form.

The understanding of real world depends on the reader's view of the universe, be it materialist, dualist, or pluralist (Popper, 1978). The real world might not be limited to the sole physical objects, but it can be extended to comprehend what Popper $(1972,1978)$ defined as World 2 and 3. It can include non-physical entities, such as concepts, theories, mythological creatures, and fictional characters. If such a non-materialist view is taken into account, then for instance despite zombies might not be real, they are part of the real world as part of Popper's World 3 (human knowledge). Otherwise, if a materialist view is considered, the real world will just include the physical documents describing those non-physical entities. In this case, 'zombies' is part of the reality just as a seven-character English word.

At the other end of the spectrum, the system world is a subset of the human knowledge, as it is available to an information system. Between these two extremes (i.e., reality and the system world), there are two levels of abstraction, more complete than the system world and not so complete as the real world. It is reasonable to picture the documented world as a more complete approximation of the real world than the perceived world, because there is much information documented that remains unknown to a user. It is as well reasonable to imagine that a user can perceive information about the world which is not in the documented world, and that can be just partially documented in real-time by its device and sent to the system. Therefore, the dimension 'world' is a partially ordered set with respect to the set inclusion relationship.

Similar notions in Geographic Information Science are based on conceptual models such as the one proposed by Peuquet $(1984 ; 2002)$, where "levels progress from reality, through the abstract, user-oriented information structure, to the concrete, machine-oriented storage structure" (Peuquet, 1984, p. 69).

This new dimension is somehow related to the notion of context, that is becoming a key factor in current definitions of relevance (see e.g., Coppola et al, 2010; Dransch, 2005; Emmanouilidis et al., 2012; Huang and Gartner, 2012; Jones and Brown, 2004; Kapitsaki et al., 2009; Mizzaro and Vassena, 2011; Reichenbacher and De Sabbata, 2011), and which is included in the third dimension 'components' of the framework developed by Mizzaro (1998) and Coppola et al. (2004). Context is a vague term, which is used to refer to very different components of relevance, from a small set of physical, raw input from a device's sensors to an informational, abstract conceptualisation of the world around a user. The new dimension 'world' defines the levels of abstraction that can be used to describe the context, both physical and informational, of the relevance relation between the user's information need and the resource. However, this meaning of context is quite different from that in Mizzaro's and Coppola et al.'s frameworks, as discussed in the following.

\subsection{The 'components' dimension}

A third change that we propose is on the fourth dimension 'components'. Coppola et al. (2004, Section 3.4) noticed that further components beyond topicality are likely to be more important in the mobile use case than in the desktop one. Although they did not provide any experimental evidence of this statement (and we 
are not aware of any such a study in the literature), they proposed convincing examples and list several aspects that should be taken into account, e.g., location, trajectory, speed, etc. However, they did not further follow this direction and did not propose any structural change to the fourth dimension, but simply suggested adding the above aspects to the context. We propose more radical changes to 'components', and we extend the set of components in order to explicitly include three further components that are among the most frequently used facets of relevance, namely: user's preferences, social context, and mobility. Also, we change the term 'task' into 'activity'. Therefore, the components of relevance included in our framework are:

- topic: the subject the user is interested in;

- activity: all the activities related to the information need, ranging from those that generated the information need to those that will be pursued on the basis of the retrieved information (this component deserves some further explanations, see below);

- preferences: user's preferences related to the topic or the activity considered (e.g., Rashid et al., 2002);

- social: the social context that can influence the relevance of an entity, such as the popularity within a community or among user's connections (e.g., Mizzaro and Vassena, 2011);

- mobility: the spatio-temporal availability of the entity (e.g., location and opening hours), the spatiotemporal situation of the user (e.g., location, time schedule, and mode of transportation), available mobility infrastructure, and topological structure (e.g., Mountain and MacFarlane, 2007);

- context: everything not pertaining to the previous components, including knowledge about the physical surroundings (e.g., light level or other geographic entities in the surroundings) and informational surroundings (e.g., information contextual to the topic or activity).

The list above does not aim to provide a definition of context in itself, but to offer a set of categories, that can be used to group attributes (i.e., variables, measured properties) identified in definitions as accounting for context. Grouping context attributes in common categories defined by the listed components provides a common ground to classify and compare relevance concepts, based on which components are used and which context attribute are accounted for in each component.

As anticipated, activity deserves a couple of remarks. A first remark is that, in our opinion, there are two reasons to prefer the term 'activity' to 'task', which is the term used by Mizzaro (1998) and Coppola et al. (2004) to name the corresponding component in their frameworks (and/or to the term 'work-task situation' used by other authors, e.g., Borlund, and Schneider, 2010). First, 'activity' is more general term referring to "something which a person or group choose to do", whereas 'task' is commonly used to define a short objective-driven action, or "a piece of work to be done" (Oxford English Dictionary). Second, using the word 'activity' emphasizes a possible connection to activity theory (Kaptelinin and Nardi, 1997; 2006), which has been suggested for modelling human activities in recent studies in the fields of human-computer interaction (Nardi, 1996; Greenberg, 2001), context-aware computing (Kaenampornpan and O'Neill, 2004), locationbased services (Huang and Gartner, 2009; Hirtle et al., 2011), and geographic relevance (Reichenbacher et al, 2009). Activity theory might be useful in this scenario as well.

A second remark is that we suggest to take into account also the activities that take place before the need arises, and not only those concerning "what the user has to do with the retrieved information" (Coppola et al., 2004, p. 3) as done in the previous models. The latter seem to have an immediate and stronger consequence on relevance (e.g., it is possible to say that a document is not relevant because it is not adequate to the task/activity that has to be carried on by the user, as already suggested by Mizzaro, 1998); however, the former can be important to frame the user situation in a more complete way. For example, arriving at a previously unvisited village at dinnertime after an eight hours bicycle trip is very different from arriving after a short car trip. Other things being equal, a user on a cycling tour with some friends might prefer restaurants with bicycle parking lots, and maybe a fountain nearby, whereas a user arriving by car with her family might prefer restaurants with reserved car parking lot, easily accessible from the main street. Hence, the relevance of each restaurant is affected by the user's previous activity in both scenarios.

Finally, we highlight a distinction between this and the other dimensions, which already exists, although not explicit, in the definition given by Mizzaro (1998). In Mizzaro's definition, the components are used to qualify the abstraction levels defined in the first two dimensions ('information resources' and 'user problem'), rather than to define an independent dimension. For instance, one can qualify a perceived information need describing the topic the user is interested in, or the informational context in which the information need arise. Our position is that 'components' should be considered as a set of attributes, rather than an actual dimension. These considerations do not cause a substantial change from the previous models, but in our 
opinion help to clarify the ontological status of components.

\subsection{The new framework}

We have therefore obtained a new framework that can be understood as composed by four dimensions (i.e., 'resources', 'user problem', 'space-time', and 'world'), plus a set of attributes (i.e., 'components') that qualify how each one of the four dimensions is defined. The main differences to previous frameworks are:

- a 'space-time' dimension in place of the previous 'time' dimension,

- a new 'world' dimension, and

- a 'components' attribute set with three additional elements.

These differences are the result of the necessity to describe new concepts of relevance, particularly those related to mobile scenarios. The new framework allows us to describe the difference between the "egocentric" concept of geographic relevance proposed by Raper (2007), who discusses how users perceive the relevance of surrounding objects based on their need, and a strictly related, but more pragmatic concept of geographic relevance proposed by Reichenbacher and De Sabbata (2011), who discuss how scores can be computed based on users' query and location at the time the query is sent. These two concepts are strongly related, but different, and clear definitions and references are necessary when discussing either or both, to avoid misunderstanding and misconception. The next section provides examples of how the framework can be used to define and discuss different concepts of relevance.

\section{Analysis of relevance concepts}

To demonstrate that our framework proposed in the previous section is more general than the frameworks discussed in Section 2 and is also more expressive in describing relevance concepts, we use its structure to analyse various concepts of relevance reported in the literature. This analysis is summarised in Table 1. We take into account the notions of relevance in Geographic Information Retrieval (GIR) systems, in Mobile Information Retrieval (MIR) systems, in Location-Based Services (LBSs), in Recommender Systems (RSs), the notion of relevance by Wilson, and two notions of Geographic Relevance (GR) (Raper 2007; Reichenbacher and De Sabbata, 2011), by Raper (labelled with GRRaper in the following) and by Reichenbacher and De Sabbata (labelled with GRR\&Din the following). These relevance concepts are listed in the first column in Table 1 , and discussed in the following.

The first row in Table 1 illustrates how relevance as it is commonly implemented in GIR systems can be defined as being concerned with documents (see column 'Resource' in the table) describing geographic entities (e.g., a corpus of descriptions of natural landscapes, Derungs and Purves, 2013) and the user's query (see column 'Need' in the table); both these elements are described using the topic component (see column 'Components').

Turning to the following three rows in the table, we can note that MIR systems (see second row in the table) usually include the mobility component (Mountain and Macfarlane, 2007) and generally account for the spatio-temporal point at which the query is submitted to the system (see the 'space-time' column), e.g., providing references to relevant documents to a user exploring a national park, Mountain and Macfarlane, 2007. Relevance as it is commonly implemented in LBSs (see third row in the table) can be defined as being concerned with features and the user's query, again at the spatio-temporal point at which it is submitted to the system (e.g., providing points of interest to a user hiking in the mountains, Hauthal and Burghardt, 2012). LBSs usually account for the topic and mobility components, although in most cases the latter is restricted to user's and entities' locations in a simple geometric representation of space. Most RSs are concerned with features and user's query, focusing on topic, social, and preferences components. RSs (see fourth row in the table) usually recommend physical objects such as books and bicycles, but they can be used to recommend documents as well (e.g., scholarly articles). In this second case, it can be argued that RSs are concerned with surrogates, rather than features.

More advanced GIR and MIR systems, LBSs and RSs account for the activity and context components as well (emphasized in italics in the first four rows of the table), where the latter is usually implemented focussing mainly on its non-geographic aspects.

The fifth row in Table 1 represents Wilson's situational relevance (Wilson, 1973) (see Section 2.1), which concerns the information as the user receives it and the user's information need as she perceives it. This definition of situational relevance does not provide further indications related to the other dimensions of 
Table 1. Different concepts of relevance involving a geographic facet, and their characterization in the proposed framework.

\begin{tabular}{|c|c|c|c|c|c|}
\hline \multirow[t]{2}{*}{ Definition } & \multicolumn{4}{|c|}{ Dimensions of Relevance } & \multirow[t]{2}{*}{ Components } \\
\hline & Resource & Need & World & Space-time & \\
\hline GIR & document & query & & & $\begin{array}{l}\text { topic, activity, } \\
\text { context }\end{array}$ \\
\hline MIR & document & query & & $\begin{array}{l}\text { query } \\
\text { submission } \\
\text { location and } \\
\text { time }\end{array}$ & $\begin{array}{l}\text { topic, activity, } \\
\text { mobility, context }\end{array}$ \\
\hline LBS & feature & query & & $\begin{array}{l}\text { query } \\
\text { submission } \\
\text { location and } \\
\text { time } \\
\end{array}$ & $\begin{array}{l}\text { topic, activity, } \\
\text { mobility, context }\end{array}$ \\
\hline RS & $\begin{array}{l}\text { feature } \\
\text { (or surrogate) }\end{array}$ & query & & & $\begin{array}{l}\text { topic, activity, } \\
\text { preferences, } \\
\text { social, context }\end{array}$ \\
\hline $\begin{array}{l}\text { IR } \\
\text { Wilson (1973) }\end{array}$ & information & $\begin{array}{l}\text { perceived } \\
\text { information } \\
\text { need }\end{array}$ & & & \\
\hline $\begin{array}{l}\text { GR } \\
\text { Raper } \\
\text { Raper (2007) }\end{array}$ & entity & $\begin{array}{l}\text { perceived } \\
\text { information } \\
\text { need }\end{array}$ & $\begin{array}{l}\text { perceived } \\
\text { (egocentric) } \\
\text { or } \\
\text { documented } \\
\text { (allocentric) }\end{array}$ & $\begin{array}{l}\text { information } \\
\text { perception } \\
\text { location and } \\
\text { time }\end{array}$ & $\begin{array}{l}\text { topic, activity, } \\
\text { mobility, context }\end{array}$ \\
\hline $\begin{array}{l}\mathrm{GR}_{\mathrm{R} \& \mathrm{D}} \\
\text { Reichenbacher \& } \\
\text { De Sabbata } \\
(2011 ; 2012)\end{array}$ & feature & query & system & $\begin{array}{l}\text { query } \\
\text { submission } \\
\text { location and } \\
\text { time }\end{array}$ & $\begin{array}{l}\text { topic, activity, } \\
\text { preferences, } \\
\text { social, mobility, } \\
\text { context }\end{array}$ \\
\hline
\end{tabular}

relevance.

The context component of relevance is usually developed in its informational aspect, rather than in its geographic aspect, as the latter is understood in GR. Raper (2007) (see penultimate row in the table, GRRaper) defined GR as an extension of Wilson's (1973) situational relevance. GRRaper concerns the geographic entities as the user perceives them and the user's information need as she perceives it, at the spatio-temporal point at which the information is perceived by the user. GRRaper concerns the perceived world if the 'egocentric' attention is assumed (Raper, 2007, p. 846), which accounts for a user's perspective on relevance, considering her knowledge and point of view. GRRaper concerns the documented world if the 'allocentric' attention is assumed (Raper, 2007, p. 846), which accounts for a detached view of the situation, closer to a system's perspective on relevance (see column 'World' in the table). The elements involved in the GR relationship are described by GRRaper using the components topic, activity, mobility, and context.

A more pragmatic definition of GR has been proposed more recently by Reichenbacher and De Sabbata (Reichenbacher and De Sabbata, 2011; De Sabbata and Reichenbacher, 2012) (last row in the table, GRR\&D). This definition is similar to the concept of relevance defined by Saracevic and Kantor (1988). The latter can be described in the presented framework as the relevance of a surrogate to a user's request, with respect to the components topic, task, and context. GRR\&D concerns features and the user's information need as it is formulated in the query language, at the spatio-temporal location at which the user submits the query to the system. GRR\&D concerns the system's world, including the information already available to the system and the incoming information from the user's device. Finally, the elements involved in the GR relationship are described by GRR\&D using the components topic, activity, preferences, social, mobility, and context. For instance, an information system based on GRR\&D would evaluate the relevance of an entry in a point-ofinterest database referring to a restaurant, with respect to a query submitted by a user, considering her location and time when the query has been submitted. Moreover, an information system based on GRR\&D would take into account the information about topic, activity, preferences, social, mobility, and context, which 
is stored in the system, encoded in the query, or submitted by the sensors of the user's device.

A key difference between situational relevance as defined by Wilson (1973) and GRRaper as defined by Raper (2007) is that the former defines the relevance of a document referring to a physical entity, whereas the latter defines the relevance of a physical entity. A difference of the same kind can be found when comparing relevance as defined in GIR (Palacio et al., 2010) and MIR (Mountain and Macfarlane, 2007) (which are pragmatic derivations of situational relevance) and GRR\&D as defined by Reichenbacher and De Sabbata (Reichenbacher and De Sabbata, 2011; De Sabbata and Reichenbacher, 2012). A surrogate taken into account in IR, GIR, and MIR provides information about a document, such as an entry in database of crawled Web pages and a set of entries in an inverted index. A feature taken into account in GRR\&D provides information about a physical entity, that is a representation within an information system, such as an entry in a point-of-interest database, and linked information (e.g., a text description, or a referred Web page).

\section{Conclusions}

In this paper, we have discussed the emerging features of current concepts of relevance, which have been triggered by the rise of mobile use cases in IR. To handle these novel concepts, we proposed a conceptual framework, consisting of four dimensions and a set of components of relevance. This new framework amplifies the work of Mizzaro (1998) and Coppola et al. (2004) by (i) substituting the temporal dimension with a spatio-temporal dimension, (ii) adding a dimension holding different abstractions of reality, and (iii) revising the set of components of relevance.

The 'spatio-temporal' dimension is proposed to account for the interdependence of space and time due to users' mobility in the mobile use cases. This updated dimension allows to conceptualise the point in space and time at which a given notion of relevance refers to. The dimension 'world' is introduced due to the rising importance of the contextual component of relevance in current IR studies. This new dimension allows to conceptualise the level of abstraction taken into account by a given notion of relevance to describe the world in which the information seeking happens. Finally, the components 'mobility', 'preferences', and 'social' are added to the 'components' dimension. These three new components allow to conceptualise notions of relevance including users mobility, preferences, social context, which are currently among the most widely adopted facets of relevance.

It is our opinion that both the conceptual framework and the discussions presented above can contribute to the future development of relevance in the scope of mobile information seeking scenarios.

\section{References}

Borlund, P. (2003),"The concept of relevance in IR". Journal of the American Society for information Science and Technology, 54, 10, pp. 913-925.

Borlund, P. and Schneider, J.W. (2010), "Reconsideration of the simulated work task situation: a context instrument for evaluation of information retrieval interaction", in proceedings of the third symposium on Information interaction in context. IIIX '10, New York, NY, USA, ACM,pp. 155-164.

Cooper, W. (1971), “A definition of relevance for information retrieval”, Information storage and retrieval, 7, 1, pp. 19-37.

Coppola, P., Della Mea, V., Di Gaspero, L., and Mizzaro, S. (2004), "The concept of relevance in mobile and ubiquitous information access", in Crestani, F., Dunlop, M., Mizzaro, S., (Eds.), Mobile and Ubiquitous Information Access. Volume 2954 of Lecture Notes in Computer Science, Springer Berlin / Heidelberg, pp. 1-10.

De Sabbata, S. and Reichenbacher, T. (2012), "Criteria of geographic relevance: an experimental study", International Journal of Geographical Information Science, 26, 8, pp. 1495-1520.

Derungs, C. and Purves, R.S. (2013), "From text to landscape: locating, identifying and mapping the use of landscape features in a swiss alpine corpus", International Journal of Geographical Information Science, 28, 6, pp. 1272-1293.

Dransch, D. (2005), "Activity and context-a conceptual framework for mobile geoservices", Map-based mobile services, Springer Verlag, Berlin, pp. 31-44.

Emmanouilidis, C., Koutsiamanis, R. and Tasidou, A. (2012), “Mobile guides: Taxonomy of architectures, context awareness, technologies and applications", Journal of Network and Computer Applications, , 36, 1, pp. 103-125.

Golledge, R. (2002), "The nature of geographic knowledge", Annals of the Association of American Geographers, 92, 1, pp. 1-14.

Greenberg, S. (2001), “Context as a dynamic construct”, Human-Computer Interaction, 16, 2-4, 2, pp. 57-268.

Hägerstraand, T. (1970). "What about people in regional science?", Papers in Regional Science 24, 1, pp. 7-24.

Hauthal, E.,and Burghardt, D. (2012), "Investigation and Development of Mobile Touristic Applications", Advances in Location-Based Services, pp. 267-282.

Hirtle, S., Timpf, S. and Tenbrink, T. (2011), "The effect of activity on relevance and granularity for navigation”, Spatial Information Theory, pp. 73-89.

Huang, H. and Gartner, G. (2009), "Using activity theory to identify relevant context parameters”, Location Based Services and TeleCartography II, pp. 35-45. 
Huang, H. and Gartner, G. (2012), "Using context-aware collaborative filtering for poi recommendations in mobile guides”, Advances in Location-Based Services, pp. 131-147.

Jones, G. and Brown, P. (2004), “Context-aware retrieval for ubiquitous computing environments", Mobile and ubiquitous information access, pp. 371-374.

Kaenampornpan, M. and O’Neill, E. (2004), “Modelling context: An activity theory approach”, Ambient Intelligence, pp. 367-374.

Kapitsaki, G., Prezerakos, G., Tselikas, N. and Venieris, I. (2009), "Context-aware service engineering: A survey", Journal of Systems and Software, 82, 8, pp.1285-1297.

Kaptelinin, V. and Nardi, B. (1997), "Activity theory: basic concepts and applications" In CHI'97 extended abstracts on Human factors in computing systems: looking to the future, ACM, pp. 158-159.

Kaptelinin, V. and Nardi, B. (2006), "Activity theory in a nutshell”, Acting with Technology: Activity Theory and Interaction Design, pp. 2972.

Miller, H. (1991), “Modelling accessibility using space-time prism concepts within geographical information systems”, International journal of geographical information systems, 5, 3, pp. 287-301.

Miller, H. and Bridwell, S. (2009), "A field-based theory for time geography", Annals of the Association of American Geographers, 99, 1, pp. 49-75.

Mizzaro, S. (1997), "Relevance: The whole history”, Journal of the American Society for Information Science, 48, 9, pp. 810-832.

Mizzaro, S. (1998), "How many relevances in information retrieval?" Interacting with computers, 10, 3, pp. 303-320.

Mizzaro, S. and Vassena, L. (2011), "A social approach to context-aware retrieval”, World Wide Web, 14, 4, pp. 377-405.

Mountain, D. (2005), "Exploring mobile trajectories: An investigation of individual spatial behaviour and geographic filters for information retrieval", PhD thesis, City University, London.

Mountain, D. and Macfarlane, A. (2007), "Geographic information retrieval in a mobile environment: evaluating the needs of mobile individuals", Journal of Information Science, 33, 5, pp. 515-530.

Nardi, B. (1996), "Studying context: A comparison of activity theory, situated action models, and distributed cognition", In Context and consciousness, Nardi B. (Ed.). Massachusetts Institute of Technology, Cambridge, MA, USA, pp. 69-102.

Ostuni, V.C., Di Noia, T., Mirizzi, R., Romito, D. and Di Sciascio, E. (2012), "Cinemappy: a context-aware mobile app for movie recommendations boosted by dbpedia", in proceedings of the 1st International Workshop on Semantic Technologies meet Recommender Systems \& Big Data (SeRSy 2012), CEUR-WS.

Palacio, D., Cabanac, G., Sallaberry, C. and Hubert, G. (2010), "On the evaluation of geographic information retrieval systems", International Journal on Digital Libraries, 11, 2, pp. 91-109.

Peuquet, D. (1984), "A conceptual framework and comparison of spatial data models", Cartographica: The International Journal for Geographic Information and Geovisualization, 21, 4, pp. 66-113.

Peuquet, D. (2002).,“Representations of space and time”, Guilford Press.

Popper, K. (1972), “Objective knowledge: An evolutionary approach”, Oxford: Clarendon Press.

Popper, K. (1978), "Three worlds", The Tanner lectures on human values, 144

Raper, J. (2007), “Geographic relevance”, Journal of Documentation, 63, 6, pp. 836-852.

Raper, J., Gartner, G., Karimi, H. and Rizos, C. (2007), "A critical evaluation of location based services and their potential”, Journal of Location Based Services, 1, 1, pp. 5-45.

Rashid, A., Albert, I., Cosley, D., Lam, S., McNee, S., Konstan, J., and Riedl, J. (2002), "Getting to know you: learning new user preferences in recommender systems", in proceedings of the 7th international conference on Intelligent user interfaces, ACM, pp. 127-134.

Reichenbacher, T. (2004), "Mobile cartography: adaptive visualisation of geographic information on mobile devices”, Verlag Dr. Hut.

Reichenbacher, T. (2009), "Geographic relevance in mobile services", in proceedings of the 2nd International Workshop on Location and the Web, ACM.

Reichenbacher, T., Crease, P., and De Sabbata, S. (2009), "The concept of geographic relevance”, in proceedings of the 6th International Symposium on LBS \& TeleCartography.

Reichenbacher, T., and De Sabbata, S. (2011), “Geographic relevance: different notions of geographies and relevancies”, SIGSPATIAL Special, 3, 2, pp. 67-70.

Saracevic, T. (1975), "Relevance: A review of and a framework for the thinking on the notion in information science", Journal of the American Society for Information Science, 26, 6, pp. 321-343.

Saracevic, T., and Kantor, P. (1988), "A study of information seeking and retrieving. ii. users, questions, and effectiveness", Journal of the American Society for Information Science, 39, 3, pp. 177-196.

Saracevic, T. (2007a), "Relevance: A review of the literature and a framework for thinking on the notion in information science. Part II: nature and manifestations of relevance", Journal of the American Society for Information Science and Technology, 58, 13, pp. 19151933.

Saracevic, T. (2007b), "Relevance: A review of the literature and a framework for thinking on the notion in information science. Part III: Behavior and effects of relevance", Journal of the American Society for Information Science and Technology, 58, 13, pp. 2126-2144.

Schamber, L., Eisenberg, M. and Nilan, M. (1990), "A re-examination of relevance: toward a dynamic, situational definition", Information processing \& management, 26, 6, pp. 755-776.

Wilson, P. (1973), "Situational relevance", Information storage and retrieval, 9, 8, pp. 457-471. 